\title{
1 Differential impact of plant secondary metabolites on the soil microbiota
}

2

\section{Vadim Schütz ${ }^{1}$, Katharina Frindte ${ }^{2}$, Jiaxin Cui $^{1}$, , Pengfan Zhang $^{3}$, Stéphane}

4 Hacquard $^{3}$, Paul Schulze-Lefert ${ }^{3}$, Claudia Knief ${ }^{2}$, Margot Schulz ${ }^{1}$, Peter Dörmann ${ }^{1^{*}}$

5

$6{ }^{1}$ Institute of Molecular Physiology and Biotechnology of Plants (IMBIO), Karlrobert-Kreiten-

7 Str. 13, 53115 Bonn, Germany

$8{ }^{2}$ Institute of Crop Science and Resource Conservation (INRES), Molecular Biology of the

9 Rhizosphere, Nussallee 13, 53115 Bonn, Germany

$10{ }^{3}$ Max Planck Institute for Plant Breeding Research, Carl-von-Linne-Weg 10, 50829 Cologne,

11 Germany

12

* Correspondence:

Peter Dörmann

doermann@uni-bonn.de

Running title: Plant metabolites affect soil microbiota

Keywords: bacteria, soil, microbiota, microbiome, benzoxazinoid, benzoxazolinone, gramine, quercetin, Arthrobacter, Pseudomonas 


\section{Abstract}

24
Plant metabolites can shape the microbial community composition in the soil. Two indole metabolites, benzoxazolinone (BOA) and gramine, produced by different Gramineae species, and quercetin, a flavonoid synthesized by many dicot species, were studied for their impacts on the community structure of soil bacteria. The three plant metabolites were directly added to agricultural soil over a period of 28 days. Alterations in bacterial composition were monitored by next generation sequencing of $16 \mathrm{~S} r R N A$ gene PCR products and phospholipid fatty acid analysis. Treatment of the soil with the plant metabolites altered the composition of bacterial taxa on the phylum and genus levels. Alpha diversity was significantly altered by BOA or quercetin, but not by gramine. BOA treatment caused an increase in the relative abundances of only four genera, three of them belonging to the Actinobacteriota. Gramine or quercetin treatment resulted in the increase in relative abundance of 13 or 14 genera, respectively, most of them belonging to the Proteobacteria. The relative abundance of 22 genera was decreased after BOA treatment, 16 of which were also decreased by gramine or quercetin. Isolation and characterization of cultivable bacterial indicated an enrichment in specific Arthrobacter or Pseudomonas strains. Therefore, the effects of the treatments on soil bacteria were characteristic for each metabolite, with BOA exerting a broad-spectrum inhibitory effect, with only few genera able to proliferate, while gramine and quercetin caused the proliferation of many potentially beneficial strains. As a consequence, benzoxazolinone or gramine biosynthesis which have evolved in different barley species, is accompanied with the association with distinct bacterial communities in the soil, presumably after mutual adaptation during evolution. 


\section{$1 \quad$ Introduction}

Plants establish their specific microbial environment, starting with the attraction of

microorganisms from the soil, based on structural carbohydrates and exudates from the roots, after germination, in addition to the seed-born microbiota. Root exudates are crucial for modulating the microbial species composition in the rhizosphere to improve plant growth and the competitiveness with other organisms (Stringlis et al., 2018; Huang et al., 2019; Voges et al., 2019; Harbort et al., 2020). Metabolites and their derivatives exuded from the roots likely contribute to the adjustment of the plant microbiota and modulate its functional diversity. For example, salicylic acid exuded from willow tree roots modulates the microbiota and alters microbial gene expression in the rhizosphere (Schmidt et al., 2000; Lebeis et al., 2015). A considerable amount of allelopathic and microbiota-modifying metabolites is released from rotting plant material, and, in appropriate agricultural culture systems, also from mulches, thereby influencing the soil microbial communities in the entire agricultural ecosystem. These effects have been studied more intensively for selected metabolites, including glucosinolates and their breakdown products (isothiocyanates) (Hollister et al., 2013; Turner et al., 2013; Averill et al., 2014; Hu et al., 2014; Siebers et al., 2018; Hansen et al., 2020), isoprenoids and coumarins (Stringlis et al., 2018; Huang et al., 2019; Voges et al., 2019; Harbort et al., 2020).

Benzoxazinoids (BXs) are a class of indole-derived metabolites with allelochemical activity synthesized in Poaceae, including some Hordeum species (e.g. H. brachyantherum, H. flexuosum, H. lechleri) (Sicker et al., 2000; Grün et al., 2005). BXs, typically synthesized in young plants, have allelopathic/phytotoxic and other biocidal properties including antinutritional functions in herbivores. BXs are unstable in soil, where they are rapidly converted into benzoxazolinones (BOA, MBOA) which are detectable in the soil for up to 12 weeks. Because of their phytotoxicity, these compounds have been selected for weed control in agriculture. BOA can be degraded or detoxified by a few fungi and bacteria (Schulz et al., 2013; Schulz et al., 2017). Changes in the microbiome structure of the rhizosphere of maize plants have been studied with maize wild type and the BX-deficient bxl mutant plants. Cultivation of WT or $b x l$ plants resulted in a shift in bacterial and fungal species composition, which affected the growth of the following plant generation (Hu et al., 2018). Several bacterial and few fungal operational taxonomic units (OTUs) in the rhizosphere of maize were influenced by different $b x(b x 1, b x 2, b x 6)$ mutations (Cotton et al., 2019). Growth of the same three maize mutants led to a decline in fungal and bacterial species richness in the rhizosphere, with a decrease in many plant pathogens (Kudjordjie et al., 2019). The effects of 
BOA on microbiota composition and species diversity in the bulk soil remain, however, unclear.

Some Poaceae produce gramine as an additional indole-derived metabolite. Gramine is one of the most important allelochemicals in certain barley cultivars that do not contain BXs (Hordeum vulgare L., e.g. genotypes Lina, Osiris) (Larsson et al., 2006; Kokubo et al., 2017). Gramine is also found in other plants, for instance in lupines (Lupinus luteus L.). It affects germination and growth of oat, rye and wheat (Bravo et al., 2010). For allelopathic effects, gramine has to be released from rotting plant material, because in contrast to BXs, gramine seems to be absent from root exudates. Gramine also inhibits growth of cyanobacteria and other bacteria (Laue et al., 2014; Popp et al., 2016). The detoxification pathway of gramine in aphids (Sitobion avenae) involves carboxylesterase and glutathione S-transferase activities (Cai et al., 2004), but bacterial or plant detoxification strategies are unclear. Barley cultivars produce only one of the two metabolites, BX or gramine, indicative for divergent evolution. Therefore, it is possible that $\mathrm{BX}$ or gramine might have distinct effects on the soil microbiota. Flavonoids are polyphenols which are widespread in plants, in particular in seeds and root exudates, with quercetin representing one of the most abundant flavonoids. Flavonoids are involved in the regulation of nodulation in legumes, they exert antioxidant and antimicrobial activities, and many of them have allelopathic properties. Several flavonoids have a short lifetime while others persist in the soil, depending on the chemical structures, such as the number of functional groups, physicochemical characteristics of the soil and the rate of microbial degradation. Many Fabaceae like Lotus japonicus contain quercetin as the main flavonoid (Suzuki et al., 2008). Several microorganisms, such as Rhizobium, Agrobacterium, Pseudomonas, Bacillus and Rhodococcus species degrade flavonoids including quercetin (Pillai and Swarup, 2002). Rhizobium species detoxify flavonoids and isoflavonoids via $\mathrm{C}$ ring fission, which results in phenolic compounds like protocatechuic acid. The prooxidant activity of protocatechuic acid can cause oxidative stress and death of other microorganisms. Protocatechuic acid is also responsible for the indirect allelopathic effects of catechin, which is e.g. exuded by roots of Rhododendron formosanum (Wang et al., 2013). The rhizosphere of $R$. formosanum is rich in microorganisms that use catechin as carbon source, including Pseudomonas, Herbaspirillum and Burkholderia species. These results suggest that flavonoid breakdown products might be the true modifiers of microbial biodiversity and influence the abundance of defined species.

To study the effects of selected plant-root derived metabolites on soil microbial communities in comparison, native agricultural soil was exposed to BOA, gramine or 
quercetin, using concentrations that occur under natural conditions. We selected BOA and gramine which are two indole metabolites produced in a mutually exclusive manner by Poaceae species, to study their impact on the soil microbiome (Larsson et al., 2006). In addition, we chose to study the effects of quercetin on the soil microbiome with the aim to assess how specific the changes in the soil microbial community introduced by the two indole metabolites are. Bacterial 16S rRNA gene-based community profiling in combination with phospholipid fatty acid analyses, revealed that each of the three metabolites exerts a strong and distinct alteration in species diversity and community structure. In addition, microorganisms were retrieved from soil after application of the aforementioned plant specialized compounds with the aim of unraveling whether bacterial genera considered to be tolerant to these compounds after $16 \mathrm{~S} r R N A$ gene community profiling, can be isolated by cultivation.

\section{Materials Methods}

\subsection{Incubation of soil with secondary metabolites}

Freshly harvested, agricultural soil from Cologne (Cologne agricultural soil) (Bai et al., 2015) was sieved ( $2 \mathrm{~mm}$ mesh) and filled into open pots $(10 \mathrm{~cm}$ diameter) placed onto Petri dishes. An amount of $10 \mu \mathrm{mol}$ of solid benzoxazolinone (BOA, Fluka Chemika), gramine (SigmaAldrich) or quercetin (ABCR, Karlsruhe, Germany) was added and mixed with $300 \mathrm{~g}$ of soil (water content of $12 \% \pm 1 \%$ ). The treatment was repeated every second day over a period of 28 days. In total, $140 \mu \mathrm{mol}$ of metabolites were added to each pot equivalent to $\sim 0.5 \mu \mathrm{mol}$ per $\mathrm{g}$ soil or $\sim 3.8 \mathrm{mM}$ (based on the water content of $12 \%$ ). In addition, the soil was watered every second day with germ-free water from the top to maintain a maximal water retention capacity of $70 \%$. Control soil was watered with germ-free water without the addition of metabolites. The pots were covered with transparent plastic covers and incubated in a growth chamber under a $16 \mathrm{~h}$ light $/ 8 \mathrm{~h}$ dark cycle with $160 \mu \mathrm{mol} \mathrm{m} \mathrm{s}^{-2}$ of light, at $21{ }^{\circ} \mathrm{C}$ and $55 \%$ humidity. Four replicate pots were prepared per treatment. Soil samples $(0.5 \mathrm{~g}$ each $)$ were removed with a spatula every seven days until day 28, and placed into a Lying Matrix E tube for DNA extraction (FastDNA SPIN Kit for Soil, MP Biomedicals, Solon, USA, see below).

\subsection{Isolation and identification of cultivable bacteria from soil}


Bacterial strains were isolated from the soil after 28 days of treatment with BOA, gramine or quercetin. Soil suspensions $(1: 1, w / w)$ were prepared with sterile water, mixed and centrifuged at $2000 \mathrm{x}$ g for $15 \mathrm{~min}$. Aliquots $(50 \mu \mathrm{L})$ of a 10 -fold dilution series were plated on TSB medium (17 g/L casein peptone, $3 \mathrm{~g} / \mathrm{L}$ soya peptone, $5 \mathrm{~g} / \mathrm{L} \mathrm{NaCl}, 2.5 \mathrm{~g} / \mathrm{L} \mathrm{K}_{2} \mathrm{HPO}_{4}$, $2.5 \mathrm{~g} / \mathrm{L}$ glucose, $15 \mathrm{~g} / \mathrm{L}$ agar), YPD medium (10 g/L yeast extract, $20 \mathrm{~g} / \mathrm{L}$ bacto peptone, 20 g/L glucose, 20 g/L agar), TSM medium ( $1 \mathrm{~g} / \mathrm{L} \mathrm{K}_{2} \mathrm{HPO}_{4}, 0.2 \mathrm{~g} / \mathrm{L} \mathrm{MgSO}_{4}, 0.1 \mathrm{~g} / \mathrm{L} \mathrm{CaCl}_{2}, 0.1$ $\mathrm{g} / \mathrm{L} \mathrm{NaCl}, 0.002 \mathrm{~g} / \mathrm{L} \mathrm{FeCl}_{3}, 0.5 \mathrm{~g} / \mathrm{L} \mathrm{KNO}_{3}, 0.5 \mathrm{~g} / \mathrm{L}$ asparagine, $1 \mathrm{~g} / \mathrm{L}$ mannitol, $15 \mathrm{~g} / \mathrm{L}$ agar) and malt medium (15 g/L malt extract, $8 \mathrm{~g} / \mathrm{L}$ yeast extract, $5 \mathrm{~g} / \mathrm{L}$ glucose, $5 \mathrm{~g} / \mathrm{L}$ fructose, 10 $\mathrm{g} / \mathrm{L}$ agar). Plates were incubated for several days at $28^{\circ} \mathrm{C}$. Colonies were picked for PCR amplification of the bacterial $16 \mathrm{~S} r R N A$ gene using primers $799 \mathrm{~F}$ and $1192 \mathrm{R}$ which target the V4-V7 region. PCR products were purified by excising the $500 \mathrm{bp}$ bands after electrophoresis in $1.2 \%$ agarose gels. DNA was eluted using the NucleoSpin Gel and PCR Clean-up Kit (Macherey-Nagel). After Sanger sequencing (GATC Biotech, Konstanz, Germany), sequences were searched against the nucleotide database at Genbank using BLASTN. Obtained isolates were stored at $-80{ }^{\circ} \mathrm{C}$ in microbanks. The $16 \mathrm{~S}$ rRNA gene sequences of the isolated strains were quality checked and trimmed before they were aligned using the SINA aligner v1.2.11 (Pruesse et al., 2012) and imported into the SSU Ref NR 99138.1 database. The alignment was manually controlled and a phylogenetic tree was calculated using the maximum-likelihood algorithm PHYML in ARB (Ludwig et al., 2004).

\subsection{Bacterial community profiling by $16 \mathrm{~S} r R N A$ amplicon sequencing}

Total DNA was extracted from the soil using the FastDNA SPIN Kit for Soil (MP

Biomedicals, Solon, USA). To the soil in the Lysing Matrix E tube, sodium phosphate buffer and MT buffer were added, and it was homogenized with 2 × 30 s rotations at $6000 \mathrm{rpm}$ in a Precellys tissue homogenizer (Bertin, Frankfurt a. M., Germany). Further extraction steps were performed as described in the manufacturer's protocol. Genomic DNA from soil was eluted in $60 \mu \mathrm{L}$ of nuclease free water and purified twice using Agencourt MPure XP beads (Beckman-Coulter, Krefeld, Germany). The purified genomic DNA was used in a two-step PCR procedure to amplify the V4-V7 region of the bacterial 16S rRNA gene (primers 799F 1192R, Supplementary Table S1) (Bodenhausen et al., 2013). In the first step, the gene fragment was amplified for each sample in triplicates in a $25 \mu \mathrm{L}$ reaction volume (Agler et al., 2016). Each reaction contained 0.2 $\mu \mathrm{M}$ of each primer, GoTaq Reaction Buffer (1.5 mM $\mathrm{MgCl}_{2}$ ), PCR Nucleotide Mix (0.2 mM each dNTP), 1.25 U GoTaq G2 DNA polymerase (Promega, Walldorf, Germany), $0.3 \%$ bovine serum albumine, 4 ng template DNA and 
nuclease-free water. The first PCR amplification was performed as follows: 2 min at $94^{\circ} \mathrm{C} ; 25$ cycles of $15 \mathrm{~s}$ at $94^{\circ} \mathrm{C}, 25 \mathrm{~s}$ at $55^{\circ} \mathrm{C}, 45 \mathrm{~s}$ at $72^{\circ} \mathrm{C}$. The products were purified by electrophoresis in $1.2 \%$ agarose gels. The bands at 500 bp were excised and the DNA purified using the NucleoSpin Gel and PCR Clean-up kit (Macherey-Nagel, Düren, Germany). DNA was finally eluted in $20 \mu \mathrm{L}$ nuclease free water. The purified products were used for a second PCR, which was performed in the same way but with only 15 cycles. In the second PCR, barcoded primers containing Illumina adaptors (B5-F; B5-1 to B5-64;

Supplementary Table S1) were used. The PCR products were also purified by electrophoresis (see above). The DNA concentration of the purified PCR products was determined by fluorometry (QuantiFluor ONE Dye, Quantus Fluorometer, Promega, Walldorf, Germany), and a sequencing library was created by pooling $30 \mathrm{ng}$ of each amplicon. The library was purified twice with Agencourt MPure XP beads.

\section{$2.4 \quad$ Illumina sequencing}

The PCR products (100 ng) of the purified sequencing library were used for 2 x $300 \mathrm{bp}$ paired-end Illumina sequencing on a MiSeq sequencer (Illumina, Berlin, Germany). Raw sequence data (lllumina) were analyzed using the Quantitative Insights Into Microbial Ecology software (QIIME 1.9.0) (Caporaso et al., 2010). The taxonomic assignment is based on the Ribosomal Database Project (RDP) using a bootstrap cutoff of 0.5. An amplicon sequence variant (ASVs) table was obtained using the recently revised bacterial taxonomy system (Parks et al., 2018).

\subsection{Statistics}

The ASV table was rarefied to the lowest number of reads (7198 reads) to obtain equal read numbers for all samples. Alpha-diversity indices (Shannon, Chao1, inverse Simpson and Pilou's evenness) were calculated in R using the package vegan (Oksanen et al., 2019; R Core Team, 2020). Overall differences between groups of samples were assessed using nonparametric Kruskal-Wallis tests while Mann-Whitney tests were applied for individual comparisons, because data were non-normally distributed. A Bonferroni-Holm correction was performed to correct for multiple comparisons. The effects of treatment (control, BOA, gramine and quercetin) and sampling time points (t7, t14, t21 and t28) were evaluated.

A non-metric multidimensional scaling (NMDS) plot based on Bray-Curtis distances was built with Hellinger transformed data. To test for significant differences in the bacterial community composition in dependence on treatment and time, Permutational ANOVA 
217 (ADONIS) was performed using the Bray-Curtis distance matrix data, which were derived

218 from Hellinger transformed ASV tables (package 'vegan' version 2.5-6 in R).

219 Responsive taxa significantly enriched in relative abundance due to sample treatment 220 were identified at phylum and genus levels using ANOVA with Tukey-Kramer Post-hoc 221 analyses and a Benjamini-Hochberg false discovery rate correction in STAMP (Parks et al., 222 2014). A heatmap was generated in R using the packages pheatmap and dplyr (Kolde, 2019;

223 Wickham et al., 2020), which shows the preferential occurrence of the most abundant ASVs

224 (>0.5\% relative abundance) in dependence on treatment and time point. Data were $\log 10$

225 transformed. Dendrograms were calculated using the WPGMA clustering algorithm based on 226 an Euclidean distance matrix derived from relative ASV abundance.

227

\subsection{Phospholipid fatty acid analysis}

Lipids were extracted from the soil with chloroform/methanol. Phospholipids were isolated by solid phase extraction, fatty acids were converted into their methyl derivatives in the presence of the internal standard tridecanoic acid (13:0) and quantified by gas chromatography-mass spectrometry as described (Siebers et al., 2018).

\section{Results}

\subsection{Responses of the soil microbiota to plant metabolites}

We incubated agricultural soil in open pots with addition of one of the three plant metabolites, BOA, gramine or quercetin (each with a total amount of $\sim 0.5 \mu \mathrm{mol}$ per g soil) over a period of 28 days. Different alpha diversity indices (Shannon, Chao1, Pilou's Evenness and inverse Simpson index) as measures for biodiversity, species richness, equal distribution of species within the community and probability for the species identity of two randomly selected individuals, respectively, were calculated (Figure 1). All alpha diversity indices showed a significant reduction after BOA and quercetin treatment compared to the control. In contrast, alpha diversity indices of gramine-treated samples were not significantly different from control samples. Changes in all alpha diversity indices over time were inconsistent and rarely significant, indicating no strong alterations during the course of incubation. 

2A). A clear clustering according to treatment was evident, while temporal differences explained less of the variation in community composition between samples. These observations were confirmed by ADONIS with higher $\mathrm{R}^{2}$-values for treatment then for time points (treatment, $\mathrm{R}^{2}=0.46, P=0.001$; time point, $\mathrm{R}^{2}=0.10, P=0.001$; autocorrelation treatment $*$ time point, $\left.\mathrm{R}^{2}=0.18, P=0.001\right)$. The samples treated with $\mathrm{BOA}$, gramine or quercetin were located in separate clusters at all time points. For the control samples, the early time points $(\mathrm{t} 7, \mathrm{t} 14)$ were separated from the late ones $(\mathrm{t} 21, \mathrm{t} 28)$ which partially overlapped with the BOA treated samples. This indicates that the bacterial community structure was strongly altered by gramine and quercetin treatment, while the treatment with BOA resulted in a transient difference in the bacterial community composition compared to the control samples.

262

\subsection{Bacterial taxa are differentially affected by plant metabolites}

The phyla of Actinobacteriota, Chloroflexi and Proteobacteria were most abundant in the control soil samples with mean relative abundances of $31.9 \%, 12.1 \%$ and $46.2 \%$ at the time point t28, respectively, while Acidobacteriota, Bdellovibrionota, Firmicutes, Gemmatimonadota, Nitrospirota, Patescibacteria, unclassified bacteria and others were much less abundant (all $<2 \%$, Figure 2B). Exposure to the plant metabolites revealed clear changes in taxonomic composition. Incubation with BOA resulted in a significant increase in the relative abundances of Actinobacteriota up to $54.2 \%$ with a concomitant decrease in Proteobacteria (39.9\%), Acidobacteriota (0.2\%), Firmicutes (0.6\%) and Chloroflexi (2.7\%) at time point t 28 compared to the control treatment. Exposure to gramine did not affect relative abundances of Actinobacteriota (23.7\%) at t28 compared to the control treatment, while Proteobacteria and Bdellivibronota were significantly more abundant with $53.5 \%$ and $0.8 \%$, respectively (Figure 2B). Quercetin treatment resulted in significantly lower relative abundances of Acidobacteriota (0.2\%) and Chloroflexi (2.2\%), and higher percentages of Proteobacteria (76.0\%) and Bdellovibrionota (1.8\%) at t28. All treatment effects were tested with ANOVA.

The treatment with the three plant-derived specialized metabolites also resulted in characteristic changes at the bacterial genus level. Fifty-two genera showed a significant increase in relative abundance in response to the treatment with BOA, gramine or quercetin (all time points aggregated) (Supplementary Table S2). The genera with relative abundances $>0.5 \%$ at any time point in the control or one of the three treatments were included in a 
284 heatmap showing the changes in relative abundances between treatments and time points

285 (Figure 3). Clustering of the samples reflected NMDS results with a clear separation of 286 samples according the three treatments with the plant metabolites. Early time points of the control clustered most closely to the gramine treatment, while late control time points were most similar to the BOA treatment.

Genera with a significant increase or decrease in relative abundance after treatment with the metabolites are presented in two Venn diagrams (Figure 4). Three genera of Actinobacteriota (Arthrobacter, Pseudarthrobacter, Paenarthrobacter) and one of Proteobacteria (Cupriavidus), showed an increase in relative abundance after BOA treatment. The relative abundance of Pseudarthrobacter was also increased after gramine treatment, while Cupriavidus and Paenarthrobacter also accumulated after quercetin treatment. In addition, gramine treatment caused the accumulation of 10 different Proteobacteria genera, two genera of Bdellovibronota and one genus of Myxococcota. The genera that increased in relative abundance upon quercetin treatment were mostly Proteobacteria (9 genera), Actinobacteriota and Bdellovibrionota. Many genera decreased in relative abundance, some even below the detection limit, after application of either of the three metabolites.

Supplementary Figure S1 shows the changes in relative abundances during the metabolite treatment for some selected individual ASVs. A large number of ASVs, e.g. Massilia, showed a decrease in relative abundance after the treatment with one or several metabolites, indicating that their growth was limited by the plant metabolites in comparison with other bacteria. Other ASVs like Arthrobacter ASV2 or Pseudarthrobacter, showed a gradual increase in relative abundance during metabolite treatment, and they were also increased in the control experiment, albeit to a lower extent, suggesting that these bacteria are tolerant to the metabolites. A few ASVs like Arthrobacter ASV1 showed a maximum of relative abundance after 14 and 21 days of treatment (Figure S1A). Other ASVs accumulated to very high relative abundances at least in one of the treatments, for example Arthrobacter ASV3 which was abundant during BOA application, but was also found in the other treatments (Figure S1B). A Pseudomonas ASV showed high relative abundances of $>20 \%$ at day 7 after BOA treatment and then declined, while this ASV also accumulated later in the control samples peaking at day 21. A Sphingobium ASV accumulated up to $28 \%$ only during quercetin treatment. Furthermore, an unclassified ASV from the Micrococcaceae increased to more than $30 \%$ after BOA treatment. The distinct patterns of changes in relative abundances during metabolite treatments of the individual ASVs (Figure S1) as compared with the corresponding changes on genus level (Figures 3,4) demonstrate that the ASVs show very 
diverse responses which are lost in many cases when only condensing the responses of the whole genus.

Taken together, treatment with BOA resulted in the increase in relative abundance of only four genera, three of them belonging to the Actinobacteriota, while gramine or quercetin treatment caused an increase in relative abundance of many genera, particularly members of the Proteobacteria. On the other hand, BOA treatment caused a decrease in relative abundance of a high number of genera, while some of the relative abundances were also decreased by quercetin or gramine. However, only very few genera were exclusively decreased in abundance by gramine or quercetin alone (Figure 4).

\subsection{Phospholipid fatty acid measurements of soil samples}

The analysis of the phospholipid fatty acid (PLFA) content and composition represents a valuable tool to study the microbial diversity in the soil. Phospholipids were extracted and purified from the soil after 28 days of incubation, and PLFA measured by GC-MS after conversion into methyl esters. The total amounts of PLFA were around $0.15 \mu \mathrm{g} / \mathrm{g}$ soil in all samples without significant differences (Figure 5). Because PLFA are the major components of cellular membranes, they can serve a measure for the total amount of microbial biomass in the soil. Therefore, the total amount of microbiota in the soil was not affected after the treatment with BOA, gramine or quercetin in comparison with the control. Specific PLFAs are characteristic for bacterial or fungal taxa or for cyanobacteria and eukaryotic algae (Frostegård and Bååth, 1996). The most abundant PLFA detected in the soil samples were the saturated fatty acids 16:0 and 18:0. Furthermore, odd chain (e.g. 15:0, 17:0), methyl-branched (e.g. 15:0iso, 15:0anteiso), monounsaturated (e.g. 16:1 $\Delta 9,16: 1 \Delta 7,18: 1 \Delta 11$ ) and cyclopropane (17:0cyclo) fatty acids were identified. Odd chain, methyl-branched and monounsaturated fatty acids are mainly found in bacteria, while cyclopropane fatty acids are produced in bacteria after stress (Kaneda, 1991; Alvarez-Ordóñez et al., 2008). Indeed, we observed increases in the cyclopropane fatty acid 17:0cyclo indicating that the bacteria were subjected to stress after metabolite supplementation. Fatty acids typically found in fungi like oleic acid $(18: 1 \Delta 9)$ or diunsaturated fatty acids (e.g.18:1 $\Delta 9,12)$, or in cyanobacteria/algae (triunsaturated fatty acids, 16:3 $\Delta 7,10,13 ; 18: 3 \Delta 9,12,15)$ (Frostegård and Bååth, 1996; Ibekwe and Kennedy, 1998; Kaiser et al., 2010), were of low abundance or absent from the soil. Therefore, the soil was dominated by the presence of bacterial microbiota, with very low amounts of fungi. 


\subsection{Isolation of cultivable strains after treatment of the soil with plant metabolites}

To recover microorganisms that can withstand the treatment of the soil with the plant metabolites, microorganisms were isolated from the soil harvested after $28 \mathrm{~d}$ of incubation in the presence of BOA, gramine or quercetin and from control soil. The control soil was included to demonstrate that the composition of isolates obtained from metabolite-treated soil differs from that obtained from untreated soil. Isolates were characterized after growing the strains on different media and DNA sequencing of the 16S rRNA gene. A total of 107 bacterial strains were isolated from control and metabolite-treated soil (Supplementary

Table S3). We recovered Actinobacteriota (Paenarthrobacter, Pseudarthrobacter, Arthrobacter, Nocardioides, Mycobacterium, Streptomyces), Proteobacteria (Pseudomonas, Massilia, Cupriavidus, Limnohabitans, Novosphignobium, Sphingobium, Rhizobium, Phyllobacterium) and Firmicutes (Bacillus, Paenibacillus) from control soil and from the treated soil. To assess the relationship between the different strains, the $16 \mathrm{~S} r R N A$ gene sequences were aligned together with the closest type strain sequences, and phylogenetic trees were generated (Figure 6A, B). From this analysis it became clear that many cultivable isolates can be divided into groups depending on the treatment. For example, one BOAdependent Paenarthrobacter group, and two distinct groups of the Pseudarthrobacter strains from BOA or quercetin treated soil were identified. Further branches encompassed isolates from Burkholderiales (Massilia, Cupriavidus, Limnohabitans, isolated from BOA treated soil), a Novosphingobium branch dependent on quercetin treatment, Sphingobium (BOA dependent) and a Rhizobiales group (Rhizobium, Phyllobacterium, isolated after BOA treatment) (Figure 6A, B).

\section{Discussion}

\subsection{General effects of the plant metabolites on soil bacteria}

There is accumulating evidence in recent years demonstrating that plant metabolites can change the profile of root-associated microbial communities (Stringlis et al., 2018; Huang et al., 2019; Voges et al., 2019; Harbort et al., 2020). The soil microbiota is crucial for agricultural productivity with respect to e.g. mineral recycling, water regulation and degradation of harmful molecules. A loss of species diversity and reduced abundance in the soil can therefore result in incomplete plant microbiota with reduced functions, resulting in 
decreased growth, increased susceptibility to diseases, and finally in harvest losses. Recently, Köberl et al. identified edaphic conditions, including soil $\mathrm{pH}$ and herbicide usage as important factors for soil microbial community structures in vineyards, orchards and other crop soils (Köberl et al., 2020). Agricultural practices such as continued monocultures without fallow periods can have detrimental effects on the soil microorganisms, and these effects can be caused by the release of plant metabolites into the soil.

In the present study, we focused on the effects of two plant indole metabolites, BOA and gramine, mostly produced by Gramineae species, in comparison with the flavonoid quercetin, on soil bacterial communities. Bacterial $16 \mathrm{~S} r R N A$ gene community profiling revealed that the three metabolites conveyed distinct effects on the community structure of soil-borne bacteria. These alterations are reflected by changes in alpha diversity after BOA and quercetin treatment, while alpha diversity of gramine treatment was not significantly different from control samples (Figure 1). The three metabolites caused alterations in taxonomic diversity as revealed in the NMDS plots, while BOA treatment showed in part overlapping data points with the control (Figure 2A). These alterations were also observed by changes in relative abundances at the phylum and genus levels (Figures 2, 3, 4).

Microorganisms able to cope with the plant-derived compounds can remain in a metabolic active stage and may modulate soil parameters, such as $\mathrm{pH}$, to their favor while shaping the species composition of other bacterial groups for optimization of co-existence and cooperation. Adapted microorganisms may group for acting in concert for compound detoxification and degradation. Microorganisms with these abilities obviously remain longer in the soil, even when the crop species change in subsequent cultures. These bacteria might give report about the history of soil usage, and about species particularly adaptable to defined plant metabolites. PLFA analysis demonstrated that the treatment with plant metabolites did not affect the overall content of the microbiota in the soil. Therefore, the alterations in the abundances of ASVs as derived from amplicon sequencing are correlated with absolute changes in the amounts of the corresponding bacteria. Interestingly, the fatty acid composition indicated that the soil was dominated by bacterial microbiota with very little fungal contribution. This result is in accordance with the results of amplicon sequencing because only a low number of fungal sequences were detected.

The effects of BX on bacteria of the rhizosphere were previously studied by growing BX-producing wild type (WT) or deficient ( $b x$ ) maize plants in soil (Hu et al., 2018; Cotton et al., 2019; Kudjordjie et al., 2019). The relative abundance of specific bacterial taxa was decreased in WT compared with the $b x$ mutants, and therefore was predominantly negatively 
420 affected by the presence of BXs in WT (Cotton et al., 2019; Kudjordjie et al., 2019). While it

421 is known that the microbioms of the rhizosphere and the bulk soil are essentially different

422 because the rhizosphere microbiota represents only a fraction of the bacterial biota in the bulk

423 soil, this finding is in line with results obtained here after direct BOA application to bulk soil,

424 because only four genera were increased, while 22 were decreased (Figure 4). Thus, many

425 soil-borne bacteria that do not have rhizosphere competence might not be exposed to

426 physiologically relevant concentrations of BOA by root exudation, but can get in contact with

427 BOA by agricultural practices like crop rotation or mulches. Therefore, the results obtained

428 after BOA application to soil in the present study are relevant for maintaining soil quality. No

429 effects of the plant genotypes of maize WT or $b x$ mutants on the alpha diversity of the

430 bacterial microbiota were detected (Hu et al., 2018; Cotton et al., 2019; Kudjordjie et al.,

431 2019), in contrast to the present study where we treated bulk soil directly with BOA causing

432 distinct differences in alpha diversity (Figure 1). These contrasting results might be caused by

433 different availability of BXs in the rhizosphere and in the bulk soil. The amounts of

434 metabolites added to the soil in the present work were in the range of the quantities used in

435 previous studies. For example, BXs are released in high amounts (e.g. 0.5 to $5 \mathrm{~kg} / \mathrm{ha}$ from

436 field-grown rye) into the soil (Barnes and Putnam, 1987; Reberg-Horton et al., 2005). The BX

437 amounts in rye depend on the cultivar and the age of the plants, ranging from 160 to 2000

$438 \mu \mathrm{g} / \mathrm{g}$ dry matter. Understrup and coworkers concluded that amounts of $0.003,3$ and $30 \mu \mathrm{mol}$

$439 \mathrm{BOA} / \mathrm{g}$ soil are naturally reached by root exudation or decaying plant material (Understrup et

440 al., 2005). The biotransformation of $30 \mu \mathrm{mol} \mathrm{BOA} / \mathrm{g}$ soil was not completed after 90 days and

441 even later, BOA was still detected. In phytotoxicity experiments, concentrations of 0.1 to 5.0

$442 \mathrm{mM}$ were used to study the effects of BOA on oxidative stress in mung bean (Batish et al.,

443 2006).

444 Highest amounts of gramine were found in young leaves of wild barley accessions,

445 ranging from 2.032 to $5.290 \mu \mathrm{mol} / \mathrm{g}$ fresh weight, whereas the contents in roots were lower

446 (Maver et al., 2020). Concentrations of 0.5 and $1 \mathrm{mM}$ were employed to measure the

447 phytotoxic effects of gramine on lettuce roots (Maver et al., 2020). Total quercetin in seeds of

448 Lotus japonicus amounts to $\sim 1.4 \mathrm{mg} / \mathrm{g}(\sim 4.6 \mu \mathrm{mol} / \mathrm{g})$ seeds (Suzuki et al., 2008). Considering

449 that quercetin can be released into the soil, local concentrations of $\sim 0.5 \mu \mathrm{mol} / \mathrm{g}$ soil, might

450 easily be reached. This concentration is in the same range as that of quercetin-glycoside

451 released from white clover $(0.5 \mu \mathrm{mol} / \mathrm{g}$ soil $)$, and far below the amounts of luteolin, a

452 flavonoid related to quercetin, released from peanut residues $(0.42 \mu \mathrm{g} / \mathrm{g}$, equivalent to 120

$453 \mu \mathrm{mol} / \mathrm{g}$ soil) (Carlsen et al., 2012; Wang et al., 2018). In the present study, the three 
454 metabolites were added to the soil in total amounts $\sim 0.5 \mu \mathrm{mol} / \mathrm{g}$ of soil which is in the range

455 of the naturally occurring concentrations. In fact, HPLC measurements of the contents of

456 BOA, gramine or quercetin in the soil treated for 28 days showed that the metabolites were

457 barely detectable, indicating that their actual concentrations were much lower, presumably

458 because they were bound to soil particles or degraded by microorganisms.

459

\subsection{Specific effects on soil genera after application of BOA, gramine or quercetin}

Application of BOA, gramine or quercetin to the soil resulted in the enrichment in Paenarthrobacter, Pseudarthrobacter and Arthrobacter (Figure 4). These bacteria were also highly enriched in the isolates obtained from treated soil (50 strains of a total of 107 isolates) (Figure 6A). The group of Paenarthrobacter contains 11 BOA-dependent and one graminedependent strain. The first group of Pseudarthrobacter isolates encompassed 12 strains, two strains each isolated with quercetin or gramine, and 8 BOA-dependent isolates. The second Pseudarthrobacter group contained only strains isolated after quercetin treatment (8 isolates). Arthrobacter and related Actinobacteria are wide-spread in bulk soil, are resistant to drying and starvation and can therefore live in extreme habitats (Jones and Keddie, 2006). Many Actinobacteria strains can degrade pesticides, e.g. a Paenarthrobacter isolate was found which is able to metabolize atrazine (Deutch et al., 2018). Therefore, these bacteria harbor detoxification mechanisms and can possibly also metabolize plant metabolites. abundance or were isolated after metabolite treatment, and these genera possibly also harbor pathways to degrade BOA, quercetin or gramin. For example, BOA treatment resulted in increased abundances of two Nocarioides and one Mycobacterium ASVs. Previously, isolates of Nocardioides, Variovorax and Rhodococcus were found to degrade xenobiotics like triazine (Martínková et al., 2009; Satsuma, 2010; Satola et al., 2013). Furthermore, specific isolates of Polaromonas, Ramlibacter and Rhizobacter (increased after gramine treatment) are able to degrade xenobiotics, ginsenoids (triterpene glycosides from ginseng) or natural rubber, respectively (Mattes et al., 2008; Wang et al., 2012; Kasai et al., 2017). In addition, isolates of Janibacter (Actinobacteriota), Phenylobacterium and Sphingobium (Proteobacteria) (increased with quercetin) can degrade aromatic hydrocarbons, herbicides or pesticides, respectively (Lingens et al., 1985; Cai and Xun, 2002; Zhang et al., 2009), and thus might also be able to metabolize quercetin.

Several genera, including Cupriavidus, Allorhizobium and Bradyrhizobium, Bosea, 
$488 \mathrm{BOA}$ /quercetin, gramine or quercetin, respectively (Figure 4). Furthermore, three members of 489 the Rhizobiales (two Rhizobium, one Phyllobacterium strain) were isolated from the soil after 490 BOA treatment (Figure 6B). While some members of the Rhizobiales might belong the non491 nitrogen fixing Rhizobia, many of which being associated with the root microbiome (Garrido492 Oter et al., 2018), others might belong to the nodule-forming Rhizobia which establish 493 symbiotic interactions with legumes, thereby contributing to increase the nitrogen availability 494 in the soil with beneficial effects for the plants that release these metabolites (Vandamme and 495 Coenye, 2004).

496 Furthermore, strains of Bdellivibrio, Bacteriovorax, and Peredibacter showed 497 increased relative abundance after gramine or quercetin treatment, respectively. These 498 bacteria of the Bdellovibrionota are predators feeding on susceptible bacteria (Davidov et al., 2006). The elimination of hazardous or pathogenic bacteria after attraction of Bdellovibronota members by gramine or quercetin might have beneficial effects for the plants.

Treatment with gramine or quercetin resulted in the increase in relative abundance of Novosphingobium and Massilia bacteria, and five Novosphingobium isolates were obtained after quercetin treatment. Specific strains of Novosphingobium or Massilia harbor plant growth promoting properties, e.g. auxin or siderophore production and thus, might also be beneficial for the growth of gramine- or quercetin-producing plants (Ofek et al., 2012; Rangjaroen et al., 2017).

Many Pseudomonas isolates were obtained (31 isolates of 107 strains) from control soil or after treatment with BOA, gramine, or quercetin, which might in part be due to the exceptionally high growth rate of Pseudomonas strains (Figure 6B). Only two Pseudomonas strains, QB16 and QB21, were found after quercetin treatment. A large branch contained 29 Pseudomonas isolates mostly from control, BOA-treated or gramine-treated soil. The sequence of the ASV BB20 from BOA-treated soil was different from the other Pseudomonas sequences and highly similar to that of $P$. putida KT2440, a strain which was attracted to the

515 harbors plant growth promoting properties because it leads to systemic defense priming (Neal 516 et al., 2012).

517 BOA application exerted the strongest negative impact on the abundance of bacterial

518 ASVs, with 22 genera being decreased. Many genera were specifically affected by BOA, 519 including three ASVs representing genera of Firmicutes (Paenibacillus, Geobacillus, 520 Bacillus). Members of the Bacillaceae family were also affected by BX exuded from maize 521 roots (Cotton 2019). The abundance of different Mycobacterium and Streptomyces was 
522 decreased with BOA, in line with previous results which showed that isolates from these

523 genera were sensitive to BXs (Anzai et al., 1960; Atwal et al., 1992; Schütz et al., 2019). The

524 relative abundances of Flavobacterium, Lysobacter and Nitrospira were decreased by all

525 three metabolites. Previously, quercetin was found to inhibit growth of a Flavobacterium

526 strain (Schrader, 2008), and Flavobacterium and Lysobacter strains were also affected by

527 BXs exuded from maize roots (Hu et al., 2018; Cotton et al., 2019). Nitrospira belongs to the

528 nitrifying bacteria which oxidize ammonia or nitrite to nitrate (Daims and Wagner, 2018).

529 Flavonoids with structures similar to quercetin can inhibit nitrifying bacteria, thereby

530 preventing the oxidation of ammonia in the rhizosphere resulting in a beneficial effect for

531 plant growth (Erickson et al., 2000). The abundance of only one Pseudomonas ASV was

532 specifically decreased after gramine treatment. The genus Pseudomonas encompasses many

533 soil bacteria, some of which are plant pathogens, like $P$. syringae. It is possible that gramine

534 treatment in the present study decreased the abundance of pathogenic Pseudomonas strains, in

535 agreement with previous results which showed that $P$. syringae is sensitive to gramine

536 (Sepulveda and Corcuera, 1990).

537

\section{Conclusion}

539 BOA treatment caused the increase in the relative abundance of only four genera, three of

540 them belonging to the Actinobacteriota, while 21 ASVs of different phyla were inhibited.

541 Therefore, BOA can be regarded as a broad-spectrum toxin for soil bacteria, with only few

542 genera being able to proliferate. Interestingly, the general impact of the other two metabolites,

543 gramine and quercetin, on soil bacteria, was related, because many more genera were

544 increased after gramine (14) or quercetin (13) treatment, with most genera belonging to the

545 Proteobacteria. Almost all bacterial genera inhibited by gramine or quercetin were also

546 inhibited by BOA, suggesting that these strains show a general sensitivity to toxic plant

547 metabolites rather than specifically to gramine or quercetin. Therefore, BOA on the one hand

548 and gramine/quercetin on the other hand reveal different effects on soil bacteria, with BOA

549 showing a broad-spectrum toxic effect preventing the accumulation of many presumably

550 harmful genera, while gramine and quercetin might predominantly exert their function by

551 attracting beneficial genera.

552

553

554

555 6. Contribution to the Field Statement (200 words) 
557 Plant metabolites can be exuded from the roots or incorporated into the soil from decaying

558 plant material or mulches. Specific metabolites, e.g. glucosinolates produced in rapeseed, can

559 affect the bacterial and fungal community composition in the rhizosphere and the soil.

560 Specific plants produce different metabolites, in some cases in mutually exclusive manner.

561 For example, benzoxazolinone is produced in some barley (Hordeum) species, while others

562 synthesize gramine. The capacity for benzoxazolinone and gramine synthesis is based on the

563 availability of specific genes, but the functional reason for this difference remains unclear. We

564 studied the impact of benzoxazolinone and gramine on soil bacteria, in comparison with a

565 third metabolite, quercetin, which is produced by a large group of plants. Treatment with all

566 three metabolites resulted in strong and specific alterations of soil bacteria composition. The

567 results suggest that benzoxazolinone exerted a broad-spectrum inhibitory effect on many soil

568 bacteria, in contrast to gramine and quercetin which only inhibited a few strains and favored

569 the growth of many, potentially beneficial strains. As a consequence, benzoxazolinone or

570 gramine biosynthesis which have evolved in different barley species, is accompanied with the

571 association with distinct bacterial communities in the soil, presumably after mutual adaptation

572 during evolution.

$574 \quad 7 . \quad$ Conflict of Interest

575 The authors declare that the research was conducted in the absence of any commercial or

576 financial relationships that could be construed as a potential conflict of interest.

577

\section{Author Contributions}

579 VS, MS and PD conceived the study. KF, PZ, SH, and PS-L contributed to the data analysis

580 of bioinformatics. VS, JC, PZ and SH contributed to the soil sampling. VS and JC performed

581 DNA extractions and PLFA analysis. VS and PD contributed to draft the article. KF, PS-H,

$582 \mathrm{CK}, \mathrm{MS}$ and PD contributed to critically review and edit the manuscript. All authors

583 contributed to the article and approved the submitted version.

\section{Funding}

587 Funding from the Deutsche Forschungsgesellschaft (Priority Program SPP 2125, DECRyPT, 
bioRxiv preprint doi: https://doi.org/10.1101/2021.02.09.430398; this version posted February 9, 2021. The copyright holder for this preprint (which was not certified by peer review) is the author/funder. All rights reserved. No reuse allowed without permission.

590

591

592

593

10. Acknowledgements

594 We thank Meike Siebers (University of Düsseldorf) for help with the protocol of PLFA

595 analysis.

596

\section{11. Data Availability Statement}

598 The datasets (amplicon and Sanger sequences) generated for this study can be found in the

599 NCBI Sequence Read Archive (SRA) under the BioProject accession number PRJNA699185. 


\section{Supplementary Material}

601 The Supplementary Material for this article can be found online at

602 http://www.frontiersin.org/.

603

604

Table S1. Oligonucleotides used for PCR amplification and sequencing.

605

Table S2. Bacterial genera identified in the soil after treatment with secondary

metabolites. Sequences of PCR amplicons of the $16 \mathrm{~S} r R N A$ gene were directly obtained from

soil DNA. The genera were identified by sequence comparison. The table shows mean relative frequencies and standard deviations in percent, with all time points of one treatment aggregated. Changes to the control are depicted in different colors.

Table S3. Sequences of $16 \mathrm{~S} r R N A$ amplicons from isolated cultivable bacteria. plant metabolites.

616 The changes in relative abundances of representative ASVs based on amplicon sequencing are 617 shown for the different days of treatments with BOA, gramine or quercetin.

618 A, Representative ASVs showing typical dynamics of responses to the different plant 619 metabolites.

620 B, Four ASVs that were highly abundant at least during one soil treatment. 


\section{REFERENCES}

Agler, M. T., Ruhe, J., Kroll, S., Morhenn, C., Kim, S.-T., Weigel, D., et al. (2016). Microbial hub taxa link host and abiotic factors to plant microbiome variation. PloS Biol 14, e1002352. doi: 10.1371/journal.pbio.1002352

Alvarez-Ordóñez, A., Fernández, A., Lopez, M., Arenas, R., and Bernardo, A. (2008). Modifications in membrane fatty acid composition of Salmonella typhimurium in response to growth conditions and their effect on heat resistance. Int J Food Microbiol 123, 212-219. doi: 10.1016/j.ijfoodmicro.2008.01.015

Anzai, K., Isono, K., Okuma, K., and Suzuki, S. (1960). The new antibiotics, questiomycins A and B. J Antibiot (Tokyo) 13, 125-132.

Atwal, A. S., Teather, R. M., Liss, S. N., and Collins, F. W. (1992). Antimicrobial activity of 2aminophenoxazin-3-one under anaerobic conditions. Can J Microbiol 38, 1084-1088. doi: 10.1139/m92-177

Averill, C., Turner, B. L., and Finzi, A. C. (2014). Mycorrhiza-mediated competition between plants and decomposers drives soil carbon storage. Nature 505, 543-545. doi: 10.1038/nature12901

Bai, Y., Müller, D. B., Srinivas, G., Garrido-Oter, R., Potthoff, E., Rott, M., et al. (2015). Functional overlap of the Arabidopsis leaf and root microbiota. Nature 528, 364-369. doi: 10.1038/nature16192

Barnes, J. P., and Putnam, A. R. (1987). Role of benzoxazinones in allelopathy by rye (Secale cereale L.). J. Chem. Ecol. 13, 889-906. doi: 10.1007/BF01020168

Batish, D. R., Singh, H. P., Setia, N., Kaur, S., and Kohli, R. K. (2006). 2-Benzoxazolinone (BOA) induced oxidative stress, lipid peroxidation and changes in some antioxidant enzyme activities in mung bean (Phaseolus aureus). Plant Physiol Biochem 44, 819-827. doi: 10.1016/j.plaphy.2006.10.014

Bodenhausen, N., Horton, M. W., and Bergelson, J. (2013). Bacterial communities associated with the leaves and the roots of Arabidopsis thaliana. PLoS One 8, e56329. doi: 10.1371/journal.pone.0056329

Bravo, H. R., Iglesias, M. J., Copaja, S. V., and Argandoña, V. H. (2010). Phytotoxicity of indole alkaloids from cereals. Rev. Latinoamer. Quím. 38, 123-129.

Cai, M., and Xun, L. (2002). Organization and regulation of pentachlorophenol-degrading genes in Sphingobium chlorophenolicum ATCC 39723. J Bacteriol 184, 4672-4680. doi: 10.1128/JB.184.17.4672-4680.2002

Cai, Q. N., Zhang, Q. W., and Cheo, M. (2004). Contribution of indole alkaloids to Sitobion avenae (F.) resistance in wheat. J Appl Entomology 128, 517-521. doi: 10.1111/j.1439-0418.2004.00770.x

Caporaso, J. G., Kuczynski, J., Stombaugh, J., Bittinger, K., Bushman, F. D., Costello, E. K., et al. (2010). QIIME allows analysis of high-throughput community sequencing data. Nat Methods 7, 335-336. doi: 10.1038/nmeth.f.303

Carlsen, S. C. K., Pedersen, H. A., Spliid, N. H., and Fomsgaard, I. S. (2012). Fate in soil of flavonoids released from white clover (Trifolium repens L.). Appl Environm Soil Sci 2012, 1-10. doi: $10.1155 / 2012 / 743413$

Cotton, T. E. A., Pétriacq, P., Cameron, D. D., Meselmani, M. A., Schwarzenbacher, R., Rolfe, S. A., et al. (2019). Metabolic regulation of the maize rhizobiome by benzoxazinoids. ISME J 13, 1647-1658. doi: 10.1038/s41396-019-0375-2

Daims, H., and Wagner, M. (2018). Nitrospira. Trends Microbiol 26, 462-463. doi: 10.1016/j.tim.2018.02.001

Davidov, Y., Friedjung, A., and Jurkevitch, E. (2006). Structure analysis of a soil community of predatory bacteria using culture-dependent and culture-independent methods reveals a hitherto 
undetected diversity of Bdellovibrio-and-like organisms. Environm. Microbiol. 8, 1667-1673. doi: 10.1111/j.1462-2920.2006.01052.x

Deutch, C. E., Bui, A. P., and Ho, T. (2018). Growth of Paenarthrobacter aurescens strain TC1 on atrazine and isopropylamine during osmotic stress. Ann Microbiol 68, 569-577. doi: 10.1007/s13213-018-1364-9

Erickson, A. J., Ramsewak, R. S., Smucker, A. J., and Nair, M. G. (2000). Nitrification inhibitors from the roots of Leucaena leucocephala. J Agric Food Chem 48, 6174-6177. doi: 10.1021/jf991382z

Frostegård, Å., and Bååth, E. (1996). The use of phospholipid fatty acid analysis to estimate bacterial and fungal biomass in soil. Biol Fert Soils 22, 59-65. doi: 10.1007/BF00384433

Garrido-Oter, R., Nakano, R. T., Dombrowski, N., Ma, K.-W., McHardy, A. C., and Schulze-Lefert, P. (2018). Modular Traits of the Rhizobiales root microbiota and their evolutionary relationship with symbiotic Rhizobia. Cell Host \& Microbe 24, 155-167.e5. doi: 10.1016/j.chom.2018.06.006

Grün, S., Frey, M., and Gierl, A. (2005). Evolution of the indole alkaloid biosynthesis in the genus Hordeum: distribution of gramine and DIBOA and isolation of the benzoxazinoid biosynthesis genes from Hordeum lechleri. Phytochemistry 66, 1264-1272. doi: 10.1016/j. phytochem.2005.01.024

Hansen, J. C., Schillinger, W. F., Sullivan, T. S., and Paulitz, T. C. (2020). Decline in soil microbial abundance when Camelina introduced into a monoculture wheat system. Front Microbiol 11, 571178. doi: $10.3389 /$ fmicb.2020.571178

Harbort, C. J., Hashimoto, M., Inoue, H., Niu, Y., Guan, R., Rombolà, A. D., et al. (2020). Root-secreted coumarins and the microbiota interact to improve iron nutrition in Arabidopsis. Cell Host \& Microbe 28, 825-837.e6. doi: 10.1016/j.chom.2020.09.006

Hollister, E. B., Hu, P., Wang, A. S., Hons, F. M., and Gentry, T. J. (2013). Differential impacts of brassicaceous and nonbrassicaceous oilseed meals on soil bacterial and fungal communities. FEMS Microbiol Ecol 83, 632-641. doi: 10.1111/1574-6941.12020

Hu, L., Robert, C. A. M., Cadot, S., Zhang, X., Ye, M., Li, B., et al. (2018). Root exudate metabolites drive plant-soil feedbacks on growth and defense by shaping the rhizosphere microbiota. Nat Commun 9, 2738. doi: 10.1038/s41467-018-05122-7

Hu, P., Hollister, E. B., Somenahally, A. C., Hons, F. M., and Gentry, T. J. (2014). Soil bacterial and fungal communities respond differently to various isothiocyanates added for biofumigation. Front Microbiol 5, 729. doi: 10.3389/fmicb.2014.00729

Huang, A. C., Jiang, T., Liu, Y.-X., Bai, Y.-C., Reed, J., Qu, B., et al. (2019). A specialized metabolic network selectively modulates Arabidopsis root microbiota. Science 364, eaau6389. doi: 10.1126/science.aau6389

Ibekwe, A. M., and Kennedy, A.C. (1998). Phospholipid fatty acid profiles and carbon utilization patterns for analysis of microbial community structure under field and greenhouse conditions. FEMS Microbiol Ecol 26, 151-163. doi: 10.1111/j.1574-6941.1998.tb00501.x Jones, D., and Keddie, R. M. (2006). "The Genus Arthrobacter," in The Prokaryotes: Volume 3: Archaea. Bacteria: Firmicutes, Actinomycetes, eds. M. Dworkin, S. Falkow, E. Rosenberg, K.-H. Schleifer, and E. Stackebrandt (New York, NY: Springer New York), 945-960.

Kaiser, C., Frank, A., Wild, B., Koranda, M., and Richter, A. (2010). Negligible contribution from roots

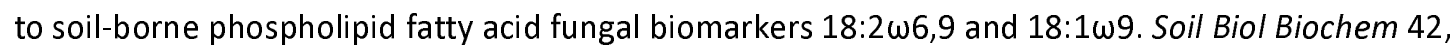
1650-1652. doi: 10.1016/j.soilbio.2010.05.019

Kaneda, T. (1991). Iso- and anteiso-fatty acids in bacteria: Biosynthesis, function, and taxonomic significance. Microbiol Rev 55.

Kasai, D., Imai, S., Asano, S., Tabata, M., Iijima, S., Kamimura, N., et al. (2017). Identification of natural rubber degradation gene in Rhizobacter gummiphilus NS21. Biosci Biotechnol Biochem 81, 614-620. doi: 10.1080/09168451.2016.1263147 
Köberl, M., Wagner, P., Müller, H., Matzer, R., Unterfrauner, H., Cernava, T., et al. (2020). Unraveling the complexity of soil microbiomes in a large-scale study subjected to different agricultural management in Styria. Front Microbiol 11, 1052. doi: 10.3389/fmicb.2020.01052

Kokubo, Y., Nishizaka, M., Ube, N., Yabuta, Y., Tebayashi, S.-I., Ueno, K., et al. (2017). Distribution of the tryptophan pathway-derived defensive secondary metabolites gramine and benzoxazinones in Poaceae. Biosci Biotechnol Biochem 81, 431-440. doi: 10.1080/09168451.2016.1256758

Kolde, R. (2019). pheatmap: Pretty heatmaps. R package version 1.0.12, https://CRAN.Rproject.org/package= pheatmap

Kudjordjie, E. N., Sapkota, R., Steffensen, S. K., Fomsgaard, I. S., and Nicolaisen, M. (2019). Maize synthesized benzoxazinoids affect the host associated microbiome. Microbiome 7, 59. doi: 10.1186/s40168-019-0677-7

Larsson, K. A. E., Zetterlund, I., Delp, G., and Jonsson, L. M. V. (2006). N-Methyltransferase involved in gramine biosynthesis in barley: cloning and characterization. Phytochemistry 67, 2002-2008. doi: 10.1016/j.phytochem.2006.06.036

Laue, P., Bährs, H., Chakrabarti, S., and Steinberg, C. E. W. (2014). Natural xenobiotics to prevent cyanobacterial and algal growth in freshwater: contrasting efficacy of tannic acid, gallic acid, and gramine. Chemosphere 104, 212-220. doi: 10.1016/j.chemosphere.2013.11.029

Lebeis, S. L., Paredes, S. H., Lundberg, D. S., Breakfield, N., Gehring, J., McDonald, M., et al. (2015). Salicylic acid modulates colonization of the root microbiome by specific bacterial taxa. Science 349, 860-864. doi: 10.1126/science.aaa8764

Lingens, F., Blecher, R., Blecher, H., Blobel, F., Eberspacher, J., Frohner, C., et al. (1985). Phenylobacterium immobile gen. nov., sp. nov., a gram-negative bacterium that degrades the herbicide chloridazon. Int J Syst Bacterio/ 35, 26-39. doi: 10.1099/00207713-35-1-26

Ludwig, W., Strunk, O., Westram, R., Richter, L., Meier, H., Yadhukumar, et al. (2004). ARB: a software environment for sequence data. Nucl Acids Res 32, 1363-1371. doi: 10.1093/nar/gkh293

Martínková, L., Uhnáková, B., Pátek, M., Nesvera, J., and Kren, V. (2009). Biodegradation potential of the genus Rhodococcus. Environ Int 35, 162-177. doi: 10.1016/j.envint.2008.07.018

Mattes, T. E., Alexander, A. K., Richardson, P. M., Munk, A. C., Han, C. S., Stothard, P., et al. (2008). The genome of Polaromonas sp. strain JS666: insights into the evolution of a hydrocarbon- and xenobiotic-degrading bacterium, and features of relevance to biotechnology. Appl Environ Microbiol 74, 6405-6416. doi: 10.1128/AEM.00197-08

Maver, M., Miras-Moreno, B., Lucini, L., Trevisan, M., Pii, Y., Cesco, S., et al. (2020). New insights in the allelopathic traits of different barley genotypes: Middle Eastern and Tibetan wild-relative accessions vs. cultivated modern barley. PLoS One 15, e0231976. doi: 10.1371/journal.pone.0231976

Neal, A. L., Ahmad, S., Gordon-Weeks, R., and Ton, J. (2012). Benzoxazinoids in root exudates of maize attract Pseudomonas putida to the rhizosphere. PLoS One 7, e35498. doi: 10.1371/journal.pone.0035498

Ofek, M., Hadar, Y., and Minz, D. (2012). Ecology of root colonizing Massilia (Oxalobacteraceae). PLoS One 7, e40117. doi: 10.1371/journal.pone.0040117

Oksanen, J., Blanchet, F. G., Friendly, M., Kindt, R., Legendre, P., McGlinn, D., et al. (2019). vegan: Community ecology package. $R$ package version 2.5-6, https://cran.ism.ac.jp/web/packages/vegan/vegan.pdf

Parks, D. H., Chuvochina, M., Waite, D. W., Rinke, C., Skarshewski, A., Chaumeil, P.-A., et al. (2018). A standardized bacterial taxonomy based on genome phylogeny substantially revises the tree of life. Nat Biotechnol 36, 996-1004. doi: 10.1038/nbt.4229 
Parks, D. H., Tyson, G. W., Hugenholtz, P., and Beiko, R. G. (2014). STAMP: statistical analysis of taxonomic and functional profiles. Bioinformatics 30, 3123-3124. doi: 10.1093/bioinformatics/btu494

Pillai, B. V. S., and Swarup, S. (2002). Elucidation of the flavonoid catabolism pathway in Pseudomonas putida PML2 by comparative metabolic profiling. Appl Environ Microbiol 68, 143151. doi: 10.1128/AEM.68.1.143-151.2002

Popp, D., Harms, H., and Sträuber, H. (2016). The alkaloid gramine in the anaerobic digestion processinhibition and adaptation of the methanogenic community. App/ Microbiol Biotechnol 100, 73117322. doi: 10.1007/s00253-016-7571-z

Pruesse, E., Peplies, J., and Glöckner, F. O. (2012). SINA: accurate high-throughput multiple sequence alignment of ribosomal RNA genes. Bioinformatics 28, 1823-1829. doi: 10.1093/bioinformatics/bts252

R Core Team (2020). R: A language and environment for statistical computing, https://www.Rproject.org/

Rangjaroen, C., Sungthong, R., Rerkasem, B., Teaumroong, N., Noisangiam, R., and Lumyong, S. (2017). Untapped endophytic colonization and plant growth-promoting potential of the genus Novosphingobium to optimize rice cultivation. Microbes Environ 32, 84-87. doi: 10.1264/jsme2.ME16112

Reberg-Horton, S. C., Burton, J. D., Danehower, D. A., Ma, G., Monks, D. W., Murphy, J. P., et al. (2005). Changes over time in the allelochemical content of ten cultivars of rye (Secale cereale L.). J. Chem. Ecol. 31, 179-193. doi: 10.1007/s10886-005-0983-3

Satola, B., Wübbeler, J. H., and Steinbüchel, A. (2013). Metabolic characteristics of the species Variovorax paradoxus. Appl Microbiol Biotechnol 97, 541-560. doi: 10.1007/s00253-012-4585-z

Satsuma, K. (2010). Mineralization of s-triazine herbicides by a newly isolated Nocardioides species strain DN36. Appl Microbiol Biotechnol 86, 1585-1592. doi: 10.1007/s00253-010-2460-3

Schmidt, S. K., Lipson, D. A., and Raab, T. K. (2000). Effects of willows (Salix brachycarpa) on populations of salicylate-mineralizing microoganisms in alpine soils. J. Chem. Ecol. 26, 2049-2057. doi: 10.1023/A:1005508230152

Schrader, K. K. (2008). Compounds with inhibitory activity against the channel catfish pathogens Edwardsiella ictaluri and Flavobacterium columnare. North Amer. J. Aquacult. 70, 147-153. doi: 10.1577/A07-027.1

Schulz, M., Marocco, A., Tabaglio, V., Macias, F. A., and Molinillo, J. M. G. (2013). Benzoxazinoids in rye allelopathy - from discovery to application in sustainable weed control and organic farming. $J$. Chem. Ecol. 39, 154-174. doi: 10.1007/s10886-013-0235-x

Schulz, M., Sicker, D., Schackow, O., Hennig, L., Hofmann, D., Disko, U., et al. (2017). 6-Hydroxy-5nitrobenzodoxazol-2(3H)-one-A degradable derivative of natural 6-Hydroxybenzoxazolin-2(3H)one produced by Pantoea ananatis. Commun Integr Biol 10, e1302633. doi: 10.1080/19420889.2017.1302633

Schütz, V., Bigler, L., Girel, S., Laschke, L., Sicker, D., and Schulz, M. (2019). Conversions of benzoxazinoids and downstream metabolites by soil microorganisms. Front. Ecol. Evol. 7, 39. doi: 10.3389/fevo.2019.00238

Sepulveda, B. A., and Corcuera, L. J. (1990). Effect of gramine on the susceptibility of barley leaves to Pseudomonas syringae. Phytochemistry 29, 465-467. doi: 10.1016/0031-9422(90)85098-Z

Sicker, D., Frey, M., Schulz, M., and Gierl, A. (2000). "Role of natural benzoxazinones in the survival strategy of plants," (Elsevier), 319-346.

Siebers, M., Rohr, T., Ventura, M., Schütz, V., Thies, S., Kovacic, F., et al. (2018). Disruption of microbial community composition and identification of plant growth promoting microorganisms 
after exposure of soil to rapeseed-derived glucosinolates. PLoS One 13, e0200160. doi: 10.1371/journal.pone.0200160

Stringlis, I. A., Yu, K., Feussner, K., Jonge, R. de, van Bentum, S., van Verk, M. C., et al. (2018). MYB72dependent coumarin exudation shapes root microbiome assembly to promote plant health. Proc Natl Acad Sci USA 115, E5213-E5222. doi: 10.1073/pnas.1722335115

Suzuki, H., Sasaki, R., Ogata, Y., Nakamura, Y., Sakurai, N., Kitajima, M., et al. (2008). Metabolic profiling of flavonoids in Lotus japonicus using liquid chromatography Fourier transform ion cyclotron resonance mass spectrometry. Phytochemistry 69, 99-111. doi: 10.1016/j.phytochem.2007.06.017

Turner, T. R., Ramakrishnan, K., Walshaw, J., Heavens, D., Alston, M., Swarbreck, D., et al. (2013). Comparative metatranscriptomics reveals kingdom level changes in the rhizosphere microbiome of plants. ISME J 7, 2248-2258. doi: 10.1038/ismej.2013.119

Understrup, A. G., Ravnskov, S., Hansen, H. C. B., and Fomsgaard, I. S. (2005). Biotransformation of 2benzoxazolinone to 2-amino-(3H)-phenoxazin-3-one and 2-acetylamino-(3H)-phenoxazin-3-one in soil. J. Chem. Ecol. 31, 1205-1222. doi: 10.1007/s10886-005-4257-x

Vandamme, P., and Coenye, T. (2004). Taxonomy of the genus Cupriavidus: a tale of lost and found. Int J Syst Evol Microbiol 54, 2285-2289. doi: 10.1099/ijs.0.63247-0

Voges, M. J. E. E. E., Bai, Y., Schulze-Lefert, P., and Sattely, E. S. (2019). Plant-derived coumarins shape the composition of an Arabidopsis synthetic root microbiome. Proc Natl Acad Sci USA 116, 12558-12565. doi: 10.1073/pnas. 1820691116

Wang, C.-M., Li, T.-C., Jhan, Y.-L., Weng, J.-H., and Chou, C.-H. (2013). The impact of microbial biotransformation of catechin in enhancing the allelopathic effects of Rhododendron formosanum. PLoS One 8, e85162. doi: 10.1371/journal.pone.0085162

Wang, H.-W., Tang, M.-J., Su, C.-L., Zhang, W., Xu, R.-S., Guan, Y.-X., et al. (2018). The alleopathic compound luteolin from peanut litter affects peanut nodule formation and the rhizosphere microbial community. Agron J 110, 2587-2595. doi: 10.2134/agronj2018.03.0149

Wang, L., An, D.-S., Kim, S.-G., Jin, F.-X., Kim, S.-C., Lee, S.-T., et al. (2012). Ramlibacter ginsenosidimutans sp. nov., with ginsen oside-converting activity. J Microbiol Biotechnol 22, 311315. doi: $10.4014 / j m b .1106 .06041$

Wickham, H., François, r., Henry, L., and Müller, K. (2020). dplyr: A grammar of data manipulation. $R$ package version 1.0.2.

Zhang, G.-Y., Ling, J.-Y., Sun, H.-B., Luo, J., Fan, Y.-Y., and Cui, Z.-J. (2009). Isolation and characterization of a newly isolated polycyclic aromatic hydrocarbons-degrading Janibacter anophelis strain JY11. J Hazard Mater 172, 580-586. doi: 10.1016/j.jhazmat.2009.07.037 
Figure Captions

Figure 1. Box plots showing differences in alpha-diversity measures of the soil bacterial communities in dependence on treatments with different plant metabolites (left column) and over time (right column). and inverse Simpson index (D). Significant differences between groups of samples are indicated by lower-case letters according to Mann-Whitney tests.

Figure 2. Changes in the soil bacterial community structure after the treatment with BOA, gramine and quercetin and at the different sampling time points.

(A) Differences in bacterial community structure between samples illustrated in non-metric multidimensional scaling (NMDS) plots. The time points are shown by different symbols, and the color code depicts the different treatments. (B) Relative abundance of bacterial phyla in the soil of the control or after treatment with BOA, gramine or quercetin. Low abundant groups with $<0.5 \%$ of the total reads are summarized as "Other". $(n=4$, mean $\pm S D)$.

Figure 3. Heatmap showing differences in relative abundances of abundant $(>0.5 \%)$ bacterial genera in soil after application of different plant metabolites over time.

Differences in relative abundance (log10-transformed) are color-coded with blue colors indicating high relative abundances, and yellow colors indicating low abundances. The dendrogram was derived from WPGMA clustering of an Euclidean distance matrix.

Figure 4. Venn diagrams showing genera with increased or decreased relative abundance after treatment with BOA, gramine or quercetin in comparison to the control.

871 Different colors indicate the assignment to the different phyla.

Figure 5. Phospholipid fatty acid (PLFA) analysis of soil samples. extracted and phospholipid fatty acids determined by GC-MS. Data are shown in \%. The inset shows total PLFA in $\mu$ g per g soil. 10:0, decanoic acid; 12:0, lauric acid; 14:0iso, 11-methyl-

877 tridecanoic acid; 15:0iso, 13-methyl-myristic acid; 15:0anteiso, 12-methyl-myristic acid; 
878 16:0iso, 13-methyl-pentadecanoic acid; 16:1 $\Delta 9$, palmitoleic acid; 16:1 $\Delta 7, \Delta 7$-hexadecenoic

879 acid; 16:0, palmitic acid; 17:0iso, 15-methyl-palmitic acid; 17:0cyclo, 7,8-cyclopropane-

880 palmitic acid; 18:1 $\Delta$, oleic acid; 18:1 $\Delta 11$, vaccenic acid; 18:0, stearic acid. (ANOVA, post

881 hoc Tukey; $\mathrm{p}<0.05 ; \mathrm{n}=3$; mean $\pm \mathrm{SD}$; different letter indicate significant differences).

882

883

Figure 6. Phylogenetic relationship of bacterial isolates obtained after treatment of the

884 soil with BOA, gramine or quercetin and from control soil.

885 The tree was calculated based on the maximum-likelihood algorithm. Color coding indicates

886 isolates from soils treated with BOA, gramine or quercetin or from control soil.

887 (A) Actinobacteria (Paenarthrobacter, Pseudarthrobacter, Arthrobacter, Nocardioides,

888 Mycobacterium, Streptomyces).

889 (B) Proteobacteria (Pseudomonas, Massilia, Cupriavidus, Limnohabitans, Novosphingobium,

890 Sphingobium, Rhizobium) and Firmicutes (Bacillus, Paenibacillus)

891

892 
bioRxiv preprint doi: https://doi.org/10.1101/2021.02.09.430398; this version posted February 9, 2021. The copyright holder for this preprint (which was not certified by peer review) is the author/funder. All rights reserved. No reuse allowed without permission.

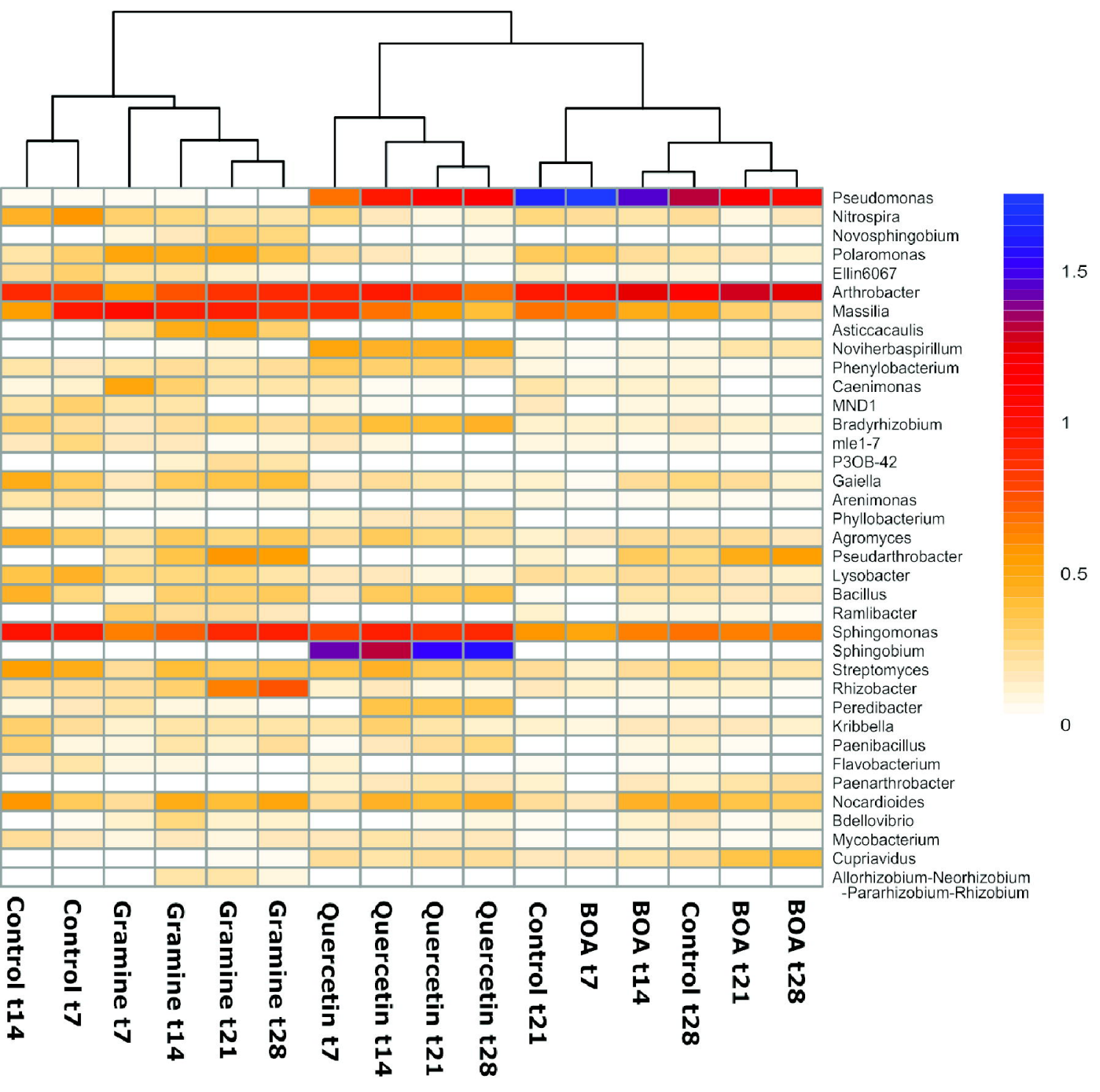

Figure 3 
bioRxiv preprint doi: https://doi.org/10.1101/2021.02.09.430398; this version posted February 9, 2021. The copyright holder for this preprint (which was not certified by peer review) is the author/funder. All rights reserved. No reuse allowed without permission.

A

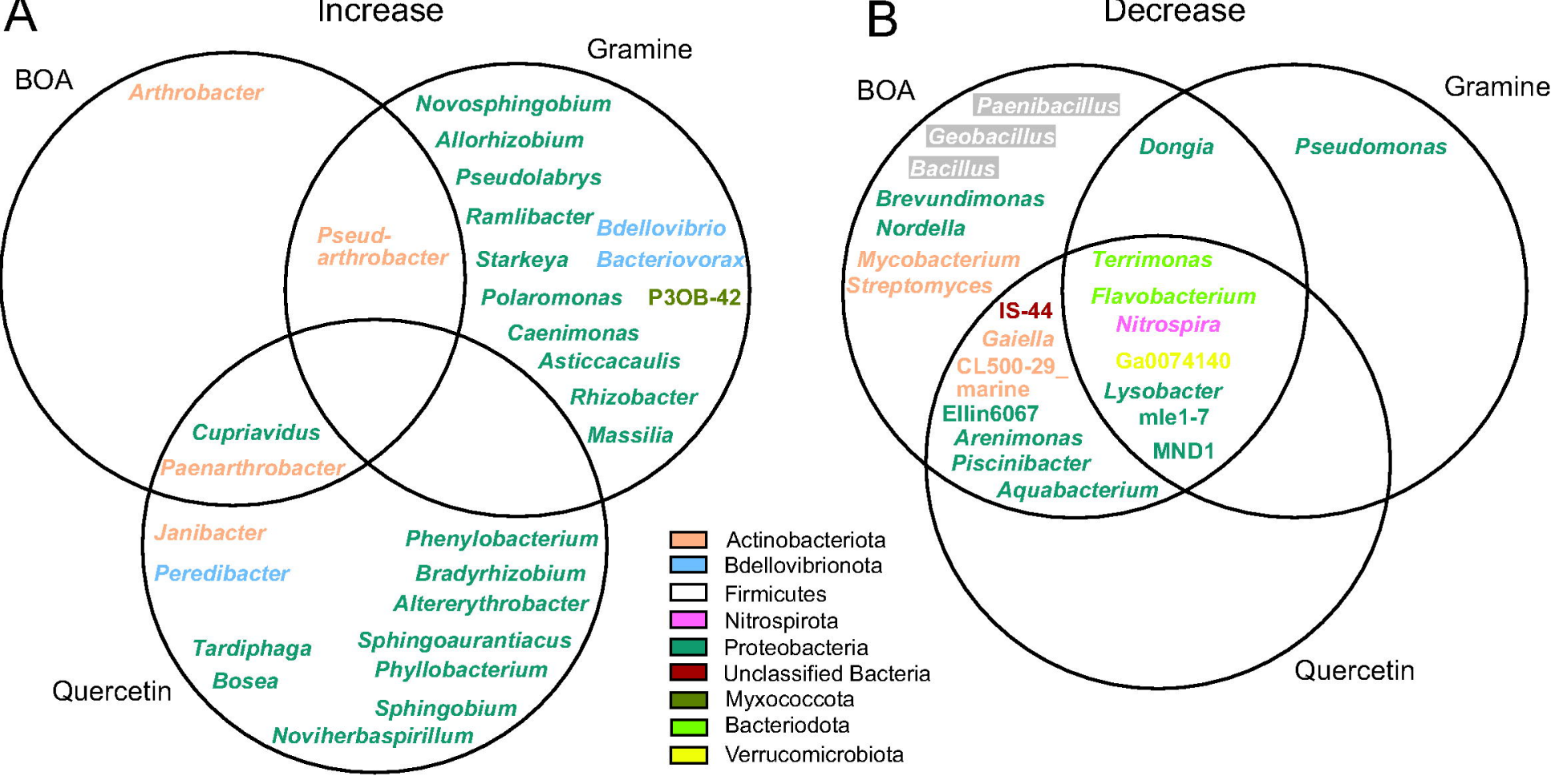

Figure 4 
bioRxiv preprint doi: https://doi.org/10.1101/2021.02.09.430398; this version posted February 9, 2021. The copyright holder for this preprint (which was not certified by peer review) is the author/funder. All rights reserved. No reuse allowed without permission.

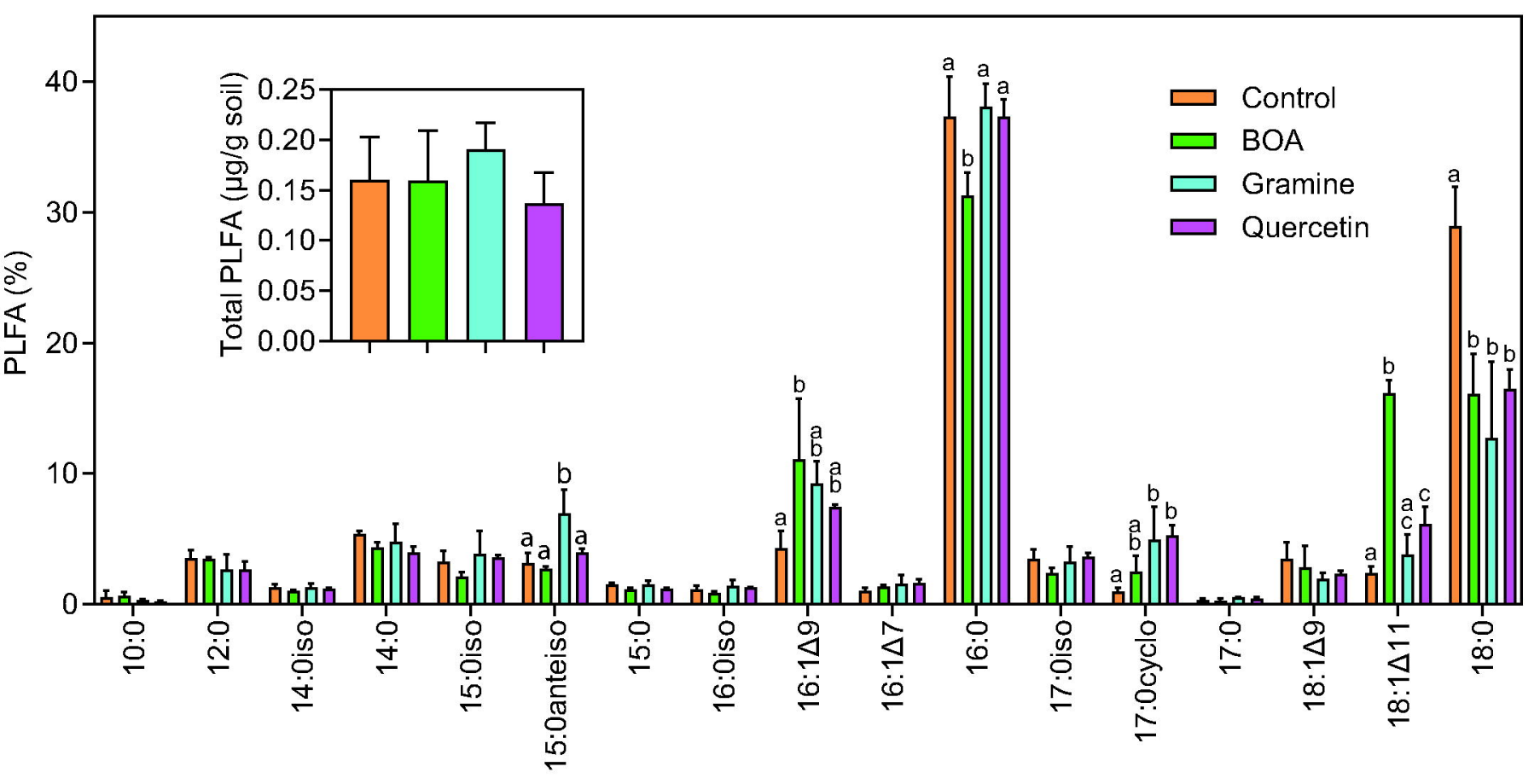

Figure 5 
bioRxiv preprint doi: https://doiforg/10.1 101/2021.02.09.430398; this ver\$ion posted February 9, 2021. The copyright holder for this preprint (which was not certffied by peer review) is the author/funder. All jights reserved. No reuse allowed without permission.

Paenarthrobacter nicotinovorans

Paenarthrobacter aurescens

Paenarthrobacter ilicis

BB12

QB14

QB18

Pseudarthrobacter sulfonivorans

Pseudarthrobacter polychromogenes

- BB34

$\mathrm{BB} 40$

$\mathrm{BB} 30$

$\mathrm{BB} 13$

B GB1

BB25

BB2

BB18

QB2

QB4

QB3

QB6

QB7

QB13

QB5

QB12

Pseudarthrobacter defluvii

$\leftarrow$ CB19

Arthrobacter humicola

Arthrobacter oryzae

- Arthrobacter cupressi

Arthrobacter globiformis NBRC 12137

Arthrobacter globiformis

Arthrobacter pascens

GB17

CB16

CB22

QB23

CB4

CB14

GB22

Pseudarthrobacter

(23)

QB17

QB1

Arthrobacter agilis

Arthrobacter methylotrophus Nocardioides daedukensis - QB10

- Nocardioides jensenii

- Nocardioides mesophilus BB38

Nocardioides maradonensis

- BB43

$[$ Mycobacterium canariasense

$L$ Mycobacterium smegmatis

Mycobacterium tuberculosis $H 37 R v$

Mycobacterium tuberculosis H37Rv

CB 21

Streptomyces antibioticus

- Streptomyces rishiriensis

Streptomyces lincolnensis

Streptomyces canus

4 Streptomyces prunicolor

$\leftarrow$ Streptomyces yangpuensis

-1 BB39

- CB24

CB27

Streptomyces pratensis

Streptomyces rhizosphaerihabitans

Streptomyces fildesensis

Streptomyces lushanensis

- B B28

Streptomyces fradiae

Streptomyces albus

Streptomyces

(20)
Arthrobacter

(26)

Nocardioides

(6)

\section{Mycobacterium \\ (6)}

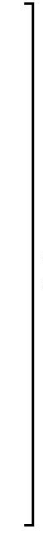

]

Proteobacteria \& Firmicutes

Figure $6 \mathrm{~A}$ 
bioRxiv preprint doi: https://doi.org/10.1101/2021.02.09.430398; this version posted February 9, 2021. The copyright holder for this preprint (which was not certified by peer review) is the author/funder. All rights reserved. No reuse allowed without permission.

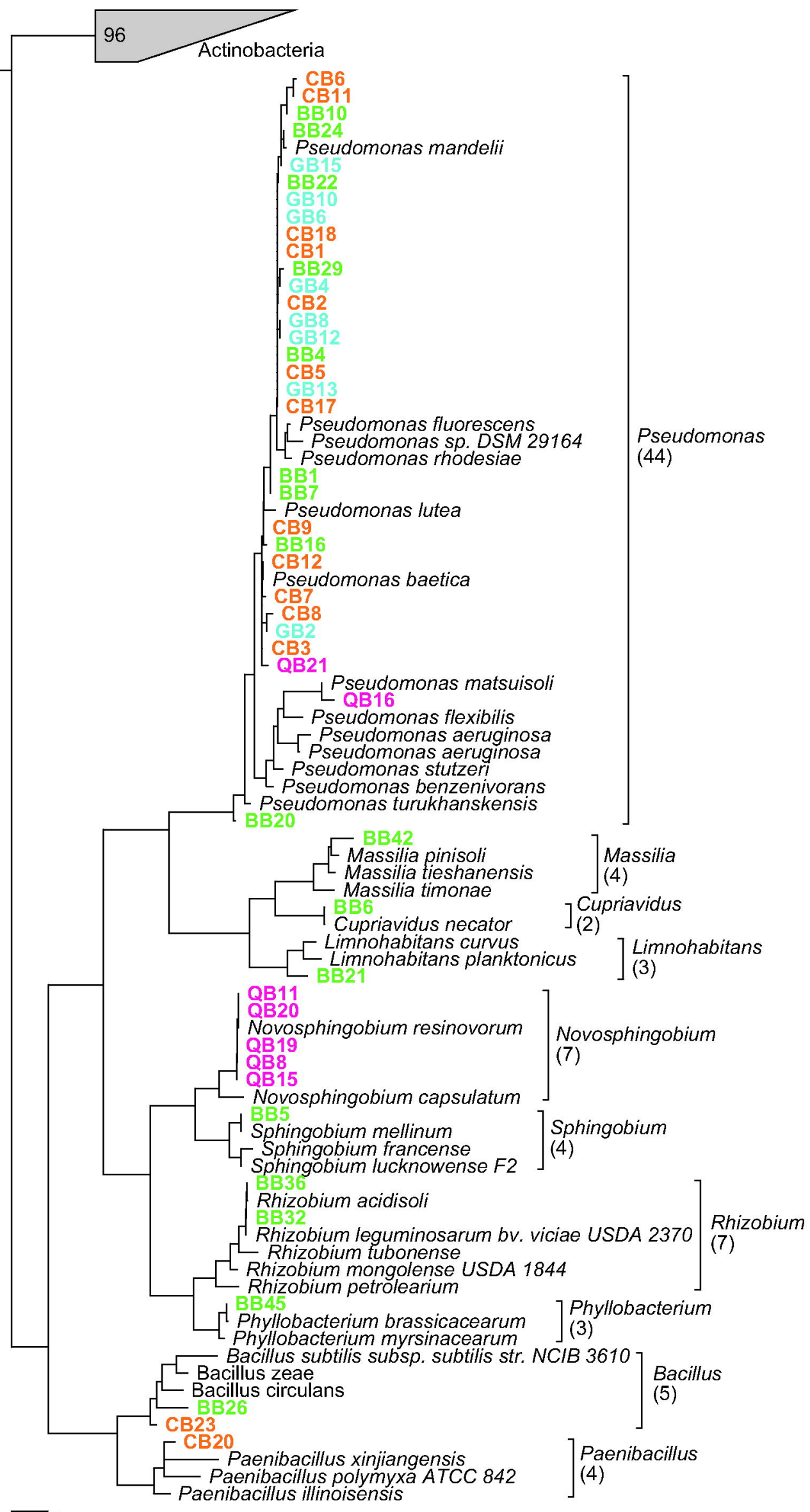

